\title{
A flexible approach
}

In the past few years, immunologists have amassed evidence to show that dendritic cells (DCs) are important sensors of the microbial environment and have a central role in the control of innate and adaptive immune responses. It is now clear that DC maturation is influenced by interaction with microbes, but can DCs distinguish different pathogens? Huang and colleagues report in Science that DCs initiate a core programme of gene regulation in response to bacterial, fungal and viral exposure, but that pathogen-specific programmes are also initiated.

To explore alterations in gene expression in DCs in response to microbial exposure they analysed the gene-expression profile of DCs in response to Escherichia coli, Candida albicans and influenza virus. Human monocyte-derived DCs were cultured with pathogens for 1-36 hours, and gene expression was analysed using an oligonucleotide array representing 6,800 genes. All three pathogens regulated the expression of a core set of 166 genes. These genes were classified according to their kinetics of expression and function. Within 4 hours of pathogen exposure, genes associated with pathogen recognition and phagocytosis were downregulated, whereas genes for antigen processing and presentation were upregulated $\sim 8$ hours post-exposure. In addition, transient increases in cytokines, chemokines and receptors involved in recruitment were observed early after exposure.

Pathogen-specific responses were also detected - the expression of 118 genes was specifically regulated by E. coli, and 58 genes by influenza. Interestingly, lipopolysaccharide, a component of E. coli, was able to regulate virtually the same set of genes as E. coli itself.

So, DCs respond to microbial exposure by regulating the expression of a core set of genes, irrespective of the stimulus, and they also exhibit pathogen-specific responses. This paper provides evidence to show that DC responses are very flexible - DCs can discriminate between pathogens and tailor their responses accordingly. But it will now be important to determine whether the responses elicited to specific pathogens are beneficial to the host or to the pathogen.

Elaine Bell

\section{Q1) References and links} ORIGINAL RESEARCH PAPER Huang, Q. etal. The plasticity of dendritic cell responses to pathogens and their components. Science 294 870-875 (2001)

FURTHER READING Reis e Sousa, C. Dendritic cells as sensors of infection. Immunity $\mathbf{1 4}$ 495-498 (2001)

ENCYCLOPEDIA OF LIFE SCIENCES Dendritic cells (T lymphocyte stimulating)

WEB SITE

Nir Hacohen's lab:

http://web.wi.mit.edu/hacohen/

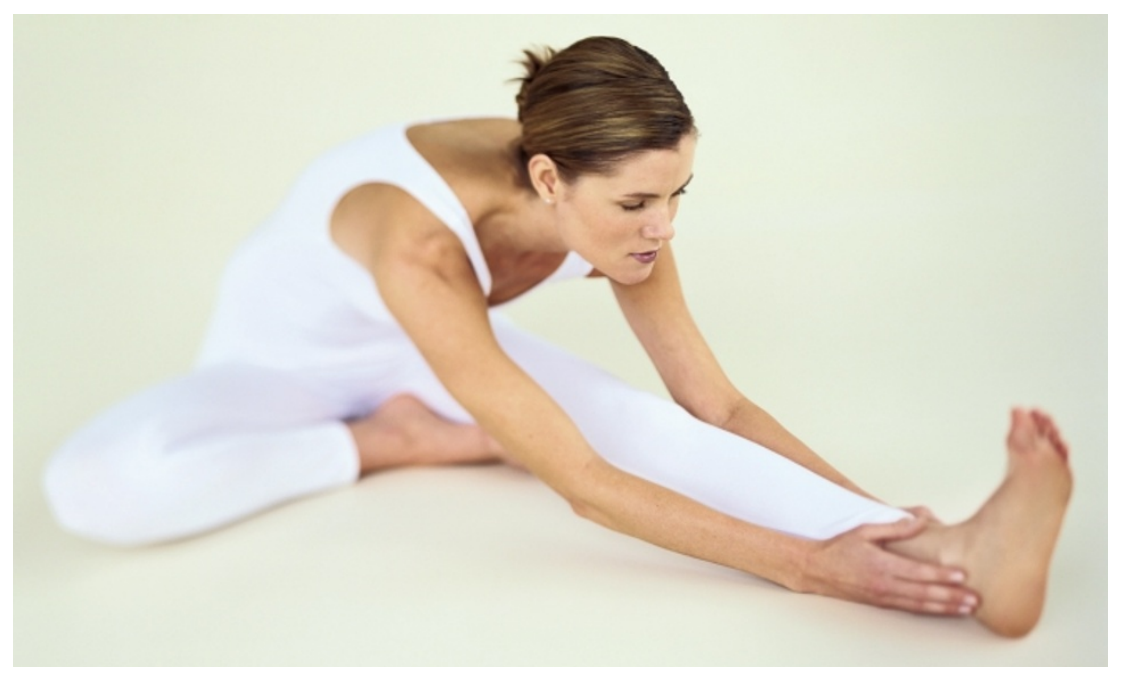

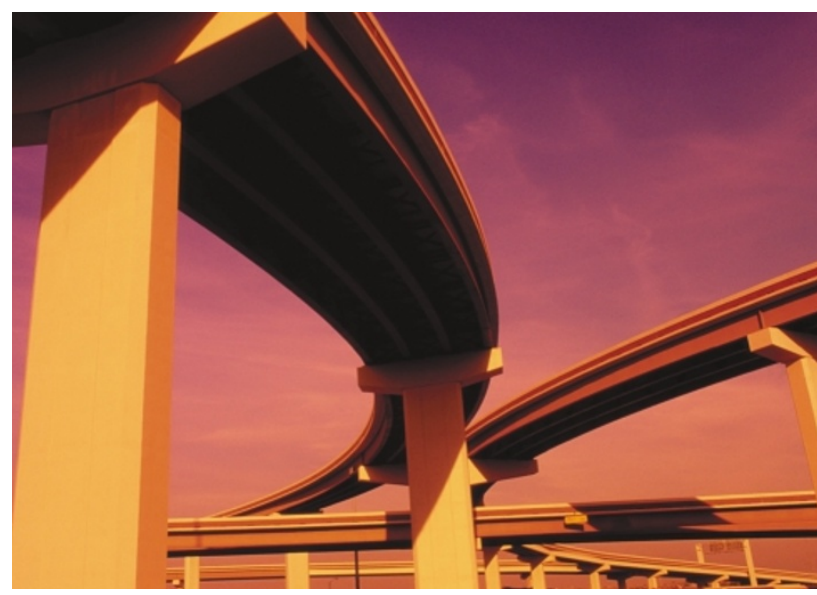

MUCOSAL IMMUNOLOGY

\section{Radical divergence}

Free radicals, such as superoxide $\left(\mathrm{O}_{2}^{--}\right)$and nitric oxide (NO), have long been thought to have deleterious roles in various inflammatory diseases. However, specificity problems associated with the use of inhibitors has made it difficult to define the precise, and often complex, pro- or anti-inflammatory roles of these molecules in experimental settings. Reporting in The Journal of Experimental Medicine, Krieglstein and co-workers used gene-targeting technology to investigate the role of free radical-producing enzymes in a mouse model of inflammatory bowel disease (IBD). Interestingly, they found diverging roles for $\mathrm{NO} \cdot$ and $\mathrm{O}_{2}^{--}$.

Using dextran sulfate sodium (DSS)-induced colitis as a model of IBD, the authors compared the response of wild-type mice with three gene-targeted mutants: mice deficient in inducible nitric oxide synthase $\left(\mathrm{iNOS}^{--}\right)$or the p47phox subunit of NADPH oxidase $\left(p 47\right.$ pho $\left.^{-/-}\right)$, or transgenic mice which overexpress superoxide dismutase (SOD). The relative protective or adverse effects of targeting these genes was determined by measuring various indicators of colitis after 7 days of DSS treatment.

The authors found that the $i N O S$-deficient mice were protected against DSS-induced colitis, whereas overexpression of SOD (which catalyses the removal of $\mathrm{O}_{2}^{--}$) was associated with exacerbated injury, and mice genetically deficient in $p 47$ phox had similar injury to the wild-type mice.

It seems that $\mathrm{NO} \cdot$ and $\mathrm{O}_{2}^{\cdot-}$ have divergent roles - $\mathrm{NO} \cdot$ proinflammatory, $\mathrm{O}_{2}^{--}$anti-inflammatory - in DDS-induced colitis. Although $\mathrm{NO}^{\cdot}$ can act beneficially as an antioxidant by scavenging peroxyl radicals and terminating lipid peroxidation, Krieglstein et al. show that it can be pro-inflammatory in certain settings. Whether $\mathrm{NO}$ contributes directly or indirectly to the inflammatory process (i.e. through the formation of peroxynitrite or perhaps nitroxyl anions) remains to be determined.

Nick Lamb

(1) References and links

ORIGINAL RESEARCH PAPER Krieglstein, C. F. et al. Regulation of murine intestinal

inflammation by reactive metabolites of oxygen and nitrogen: divergent roles of superoxide and nitric oxide. J. Exp. Med. 194, 1207-1218 (2001)

FURTHER READING Kubes, P. \& McCafferty, D. M. Nitric oxide and intestinal inflammation. Am. J. Med. 109, 150-158 (2000)

ENCYCLOPEDIA OF LIFE SCIENCES Free radicals and other reactive species in disease WEB SITE

D. Neil Granger's lab: http://www.shreveportphysiology.com/home_p26.html 